\title{
Philadelphia Chromosome
}

National Cancer Institute

\section{Source}

National Cancer Institute. Philadelphia Chromosome. NCI Thesaurus. Code C28452.

A translocation between chromosomes 9 and 22. It is the hallmark for chronic

myelogenous leukemia (CML). 